\title{
Kardio-MRT reduziert überflüssige Herzkatheter
}

Im August erschien im JAMA die erste umfassende Auswertung der CE-MARC 2 Studie aus der Arbeitsgruppe von John Greenwood, Leeds UK (Greenwood 2016). Sie zeigt, dass man bei Patienten mit stabiler Herzsymptomatik die rein diagnostischen Herzkatheter um fast 2/3 reduzieren kann, wenn man entgegen der anerkannten Praxis frühzeitig eine kardiale MRT oder eine Myokardperfusions-Szintigrafie durchführt und nach deren Ergebnis handelt. Dies steht in direktem Widerspruch zu der Risiko-adaptierten Eskalation der Diagnostik, wie sie in den meisten nationalen wie internationalen Leitlinien verankert ist.

Die CE-MARC 2 Studie zielte auf die Frage ab, mit welchem diagnostischen Pfad eine symptomatische KHK am effektivsten abgeklärt werden kann. Untersucht wurden dabei ausschließlich Patienten, die eine stabile kardial bezogene Symptomatik hatten. Die Studie randomisierte dabei die Patienten in drei diagnostische Pfade: Ein Pfad folgte den derzeit gültigen britischen
Leitlinien, die eine Eskalation der Diagnostik in Abhängigkeit von der anamnestisch zu erhebenden Pre-Test Wahrscheinlichkeit einer KHK vorsehen. Die beiden anderen Pfade nutzten als erstes diagnostisches Verfahren eine kardiale MRT oder eine Myokardiale Perufsions-Szintigrafie/MIBI SPECT (MPS) zur Abklärung einer möglichen koronaren Herzerkran-

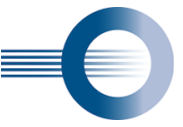

AG Herz- und Gefäßdiagnostik in der Deutschen Röntgengesellschaft

kung. Die Ergebnisse der Studie waren mit Spannung erwartet worden. Das Design dieser prospektiven, randomisierten klinischen Studie hatte die CE-MARC-2 Study Group bereits letztes Jahr im American Heart Journal publiziert (Ripley 2015).

Als erstes Ergebnis der Studie konnte Greenwood zeigen, dass bei den eingeschlossenen 1202 Patienten die Anzahl der unnötigen Herzkatheter mit 28,8 Prozent in derjenigen Gruppe am höchsten war, die nach den derzeit gültigen Leitlinien diagnostiziert wurden. Die Rate an unnötigen Herzkathetern in der Gruppe mit primärer kardialer MRT und in der Gruppe mit primärer MPS lag dagegen bei 7.5 Prozent bzw. 7.1 Prozent. Ein unnötiger Herzkatheter lag vor, wenn keine relevante koronare Stenose in Gefäßen bis 


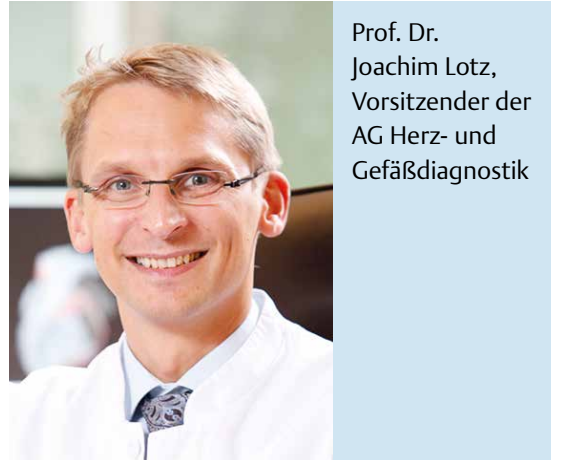

2,5 mm Durchmesser im Herzkatheter gefunden werden konnte. Die Detektion einer relevanten KHK lag bei allen drei Gruppen annähernd auf gleichem Niveau. Die Inzidenz einer schweren kardialen Komplikation (MACE) innerhalb von 12 Monaten nach initialer Diagnose war ebenfalls nicht statistisch unterschiedlich zwischen den drei Gruppen.

Die Ursache für die hohe Rate an unnötigen Herzkathetern in der Leitliniengruppe vermuten Greenwood et al. in den Bewertungsmaßstäben, mit denen die Wahrscheinlichkeit einer relevanten KHK abgeschätzt wird: Die hier verwendeten Duke-Kriterien haben ihre Wurzeln in Erkenntnissen von vor über 30 Jahren und sind nur unwesentlich an die sich verändernde Demografie, die deutlich differenzierteren Risikofaktoren sowie die diagnostischen Möglichkeiten der heutigen kardiovaskulären Medizin angepasst worden. Bereits 2011 hatte Cheng publiziert, dass die Duke-Kriterien die Inzidenz einer relevanten KHK systematisch überschätzen (Cheng 2011).

Die CE-MARC 2 Studie ist explizit nicht geeignet für die Beantwortung der Frage, ob die MRT der MPS in der Diagnostik der KHK überlegen ist. Das wurde in einer früheren Studie der gleichen Arbeitsgruppe, dem ersten CE-MARC-Trial (Greenwood 2014 , Greenwood 2012), gezeigt und von anderen Arbeitsgruppen wie der IMPACTII Studie (Schwitter 2012) bzw. einer größeren Meta-Analyse von 2015(Takx 2015) detailliert untersucht. Quintessenz dieser Studien war bislang, dass die MRT den gleichen negativen prädiktiven Wert wie die MPS zeigt, aber die tendenziell stabilere Methode ist und eine vorliegende KHK genauer zu charakterisieren vermag.

Die Ergebnisse der CE-MARC 2 Studie untermauern die diagnostische Effizienz, die kardiale MRT und MPS primär in der Abklärung kardialer Symptomatik einzuset- zen. Sie stärken damit auch den Ruf, vor allem die kardiale MRT in den Leistungskatalog der deutschen kassenärztlichen Versorgung aufzunehmen. Bislang existiert die kardiale MRT nicht im Leistungskatalog des EBM.

Die CE-MARC 2 Studie ist ein Vorbild für die Art von Studien, die notwendig sind, um neue Untersuchungsverfahren in die klinischen Handlungspfade aufnehmen zu können. Diese Studie wird bei der Überarbeitung nationaler wie internationaler Leitlinien zur Diagnostik einer koronaren Herzerkrankung die Bedeutung des frühzeitigen Einsatzes eines funktionellen, bildgestützten Verfahrens wie der MRT, MPS und perspektivisch auch der funktionellen CT-Technik stärken.

Auch diese Studie darf nicht vergessen lassen, dass wir als Ärzte den individuellen Patienten und nicht die Statistik diagnostizieren bzw. therapieren. Daher wird auf jeder Ebene des diagnostischen Pfades ein erfahrener Arzt das Recht behalten müssen, das weitere diagnostische und therapeutische Vorgehen auf die speziellen Bedingungen des ihm anvertrauten individuellen Patienten anzupassen - auch unter Berücksichtigung der vor Ort verfügbaren bildgebenden Ressourcen.

\section{Literatur}

1 Cheng VY, Berman DS, Rozanski A et al. Performance of the traditional age, sex, and angina typicality-based approach for estimating pretest probability of angiografically significant coronary artery disease in patients undergoing coronary computed tomografic angiography: results from the multinational coronary CT angiography evaluation for clinical outcomes: an international multicenter registry (CONFIRM). Circulation 2011; 124: 2423-2432, 1-8.

2 Greenwood JP, Maredia N, Younger JF et al. Cardiovascular magnetic resonance and single-photon emission computed tomography for diagnosis of coronary heart disease (CEMARC): a prospective trial. Lancet 2012; 379: 453-460.

3 Greenwood JP, Motwani M, Maredia $N$ et al. Comparison of cardiovascular magnetic resonance and single-photon emission computed tomography in women with suspected coronary artery disease from the Clinical Evaluation of Magnetic Resonance Imaging in Coronary Heart Disease (CE-MARC) Trial. Circulation 2014; 129: 1129-1138.

4 Greenwood JP, Ripley DP, Berry C et al. Effect of Care Guided by Cardiovascular Magnetic Resonance, Myocardial Perfusion Scintigraphy, or NICE Guidelines on Subsequent Unnecessary Angiography Rates: The CE-MARC 2 Randomized Clinical Trial. Jama 2016; 316 : 1051-1060.
5 Ripley DP, Brown JM, Everett CC et al. Rationale and design of the Clinical Evaluation of Magnetic Resonance Imaging in Coronary heart disease 2 trial (CE-MARC 2): a prospective, multicenter, randomized trial of diagnostic strategies in suspected coronary heart disease. American heart journal 2015; 169: 17-24 e1.

6 Schwitter J, Wacker CM, Wilke N et al. Superior diagnostic performance of perfusioncardiovascular magnetic resonance versus SPECT to detect coronary artery disease: The secondary endpoints of the multicenter multivendor MR-IMPACT II (Magnetic Resonance Imaging for Myocardial Perfusion Assessment in Coronary Artery Disease Trial). Journal of cardiovascular magnetic resonance : official journal of the Society for Cardiovascular Magnetic Resonance 2012; 14 : 61.

7 Takx RA, Blomberg BA, El Aidi H et al. Diagnostic accuracy of stress myocardial perfusion imaging compared to invasive coronary angiography with fractional flow reserve meta-analysis. Circulation Cardiovascular imaging 2015; 8. 